\title{
Step and Flash Imprint Lithography: An Efficient Nanoscale Printing Technology
}

\author{
T.C. Bailey ${ }^{a}$, S.C. Johnson ${ }^{b}$, S.V. Sreenivasan ${ }^{b}$, J.G. Ekerdt ${ }^{a}$,

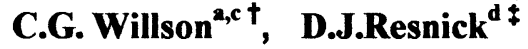 \\ ${ }^{a}$ Department of Chemical Engineering, ${ }^{b}$ Department of Mechanical Engineering, \\ ${ }^{c}$ Department of Chemistry and Biochemistry, University of Texas at Austin, Austin, TX 78759 \\ ${ }^{d}$ Motorola Labs, Tempe, AZ $85284{ }^{\dagger}$ willson@che.utexas.edu ${ }^{\ddagger}$ ayvl50@email.sps.mot.com
}

\begin{abstract}
The goal of the SFIL development program is to enable patterning of sub-100 nm features at room temperature and with minimal applied pressure. We believe the use of low viscosity materials and photopolymerization chemistry will enable SFIL to achieve the throughput required for use in the microelectronics industry. Additionally, the rigid transparent imprint template used in SFIL enables a precision in overlay alignment that is difficult to achieve in other imprint schemes. Previous work demonstrated the ability to use SFIL to pattern over existing topography, to pattern on curved surfaces, and to produce working simple optical devices. Studies of defectivity have not revealed significant defect generation, and in fact have revealed no catastrophic defect propagation. Recent work has focused on improving etching processes used to amplify the aspect ratio of the polymer features. With this recent work we have demonstrated polymer-on-Si semi-dense lines smaller than $50 \mathrm{~nm}$ made with the SFIL process.
\end{abstract}

Keywords: lithography, nanoimprint, imprint, acrylate, step and flash

\section{Introduction}

Does optical projection lithography have a limit? It surely does, but is this limit related to fundamental physics and chemistry, or rather to the cost of ownership of lithography tools? A combination of improvements in optics, reduction in wavelength, and the introduction of more complex photomasks and processes has enabled printing of features smaller than $100 \mathrm{~nm}$ by photolithography. These improvements have come at a cost, however, and this cost has increased exponentially with time. ${ }^{1}$ The Semiconductor Industry Association International Technology Roadmap for Semiconductors ${ }^{1}$ has identified alternative next generation lithography (NGL) imaging techniques based on X-ray and extreme ultraviolet (EUV) ionizing radiation, as well as techniques based on projection and direct-write electron beam lithography. Each technique has its advantages and disadvantages, but all are expensive. Many research groups are exploring alternative forms of imprint lithography, an inexpensive patterning method capable of sub- 100 $\mathrm{nm}$ resolution on various substrates. ${ }^{2-5}$

Photolithographic resolution is known to depend on the wavelength of the light, the numerical aperture of the lens, the optical system, and the resist material. ${ }^{6}$ Imprint lithography has several important advantages over conventional optical lithography and NGLs: It is non-optical by design, and the resolution appears to be limited only by the structures that can be generated in a master template. Imprint templates are typically fabricated using imaging tools such as electron beam writers that provide high resolution but lack the throughput required for mass production. By using imprint templates defined by e-beam lithography, imprint lithography takes advantage of its resolution capabilities without suffering from its throughput limitations. Although imprint equipment requires precision X-Y stages, layer-tolayer alignment systems, and wafer handling equipment common to other lithography techniques, imprint lithography realizes significant cost savings because it does not require lasers or projection optics. Since the main advances 
required to improve resolution are expected to be in the area of the imprint template development and process chemistry, an imprint tool built for one technology generation could conceivably extend to many future technology nodes.

There are many imprint lithography techniques, all variations on a common theme. The basic premise is that a template or mold with a prefabricated topography is pressed into a displaceable material. That material takes the shape of the pattern defined in the template, and through some curing process, the shaped material is hardened into a solid. The process is by nature a contact patterning process that transfers patterns without scaling. There are common challenges to all of these imprint techniques, the foremost being the dependence of this technology on 1-X imprint master resolution and the potential for defect production and propagation because of the contact nature of the replication process

\section{Background}

\subsection{Process Overview}

The SFIL process uses no projection optics and operates at room temperature. The process relies on photopolymerization chemistry and lowpressure mechanical processes to transfer patterns. There are two key differences between SFIL and other imprint lithography techniques: SFIL uses a low viscosity, photo-curable, organosilicon liquid, and SFIL uses a transparent, rigid template that allows for layer-to-layer alignment. High temperatures and imprint pressures are avoided by using a low viscosity photo-curable liquid. The transparent rigid imprint template allows flood exposure of the photopolymer to achieve cure, and enables classical optical techniques for layer-tolayer alignment.

In the SFIL process, shown in Figure 1, an organic polymer layer (transfer layer) is spincoated on a silicon substrate. A low viscosity, photopolymerizable, organosilicon solution (etch barrier) is then dispensed on the wafer in the area to be imprinted. A transparent template bearing patterned relief structures is aligned over the coated silicon substrate. The template is lowered onto the substrate, displacing the etch barrier that fills the imprint field and trapping the photopolymerizable liquid in the template relief. a)

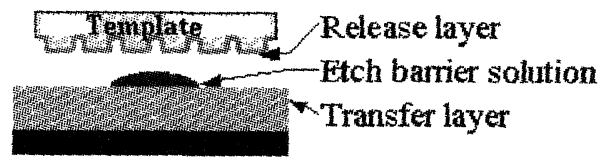

b)

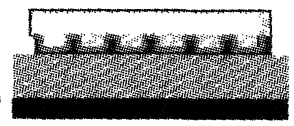

c)

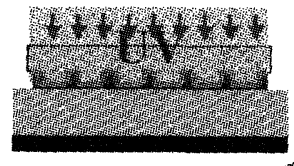

d)

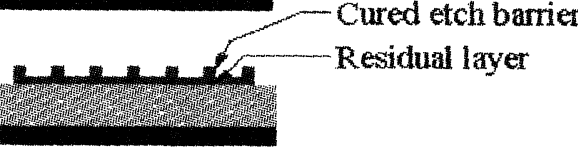

e)

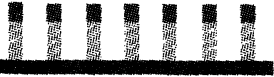

Figure 1. SFIL process flow. The process employs a template/substrate alignment scheme to bring a rigid template and substrate into parallelism (a), trapping the etch barrier (b). The gap is closed until the force that ensures a thin base layer is reached. The imprint is then illuminated through the backside of the template (c) to cure the etch barrier. The template is withdrawn (d), leaving low-aspect ratio, high resolution features in the etch barrier. The residual etch barrier (base layer) is etched away with a short halogen plasma etch, after which the pattern is transferred into the transfer layer with an anisotropic oxygen reactive ion etch (e), creating high-aspect ratio, high resolution polymer features.

Irradiation with UV light through the backside of the template cures the etch barrier into a crosslinked polymer film. A fluorocarbon release layer on the template allows separation from the substrate, leaving an organosilicon relief image that is a replica of the template pattern. A halogen etch is used to break through the undisplaced etch barrier material (residual layer) exposing the underlying transfer layer. An oxygen reactive ion etch (RIE) is used to transfer the image through the transfer layer thereby amplifying the aspect ratio of the imprinted image. The imprinting process is conducted at room temperature, and since the template is transparent, alignment schemes commonly used in mask aligners, photolithography steppers, and scanners can be implemented without difficulty. The process is simple in concept, but every step in the process presents interesting challenges in materials engineering and science. 
Careful tailoring of the chemistries of the release layer, the photopolymer formulation, and the transfer layer has allowed faithful replication of any feature on the imprint template. We have patterned areas of high and low pattern density, and produced a functional micropolarizer array with $100 \mathrm{~nm}$ Ti lines/spaces using a metal lift-off process. ${ }^{7}$ SFIL has also been used to pattern directly over a non-flat substrate, ${ }^{7}$ including curved surfaces. ${ }^{8}$ We have patterned semi-dense and isolated lines smaller than $30 \mathrm{~nm},{ }^{9}, 10$ and demonstrated the capability of layer-to-layer alignment. ${ }^{11}$

\subsection{Template Fabrication}

SFIL templates have been fabricated using the process that is similar to conventional phase-shift reticle processing. 9,10 In the standard $\mathrm{Cr}$ process, a 6 -in $\times 6$-in $\times 1 / 4$-in fused silica reticle blank coated with a thin $\mathrm{Cr}$ film is spin-coated with an electron beam resist, and baked to drive off excess casting solvent. The plate is then exposed by electrons in a direct-write e-beam tool; electron beam lithography is used because it is capable of writing very high resolution patterns. The resist is then developed, leaving a resist pattern that exposes selected portions of the underlying $\mathrm{Cr}$ film. This resist pattern is used as an etch mask to pattern the $\mathrm{Cr}$ with Cl-based RIE. The resist is then removed, leaving a $\mathrm{Cr}$ pattern on the silica substrate. A fluorine-based RIE transfers the image into the fused silica substrate to a depth of 100 to $200 \mathrm{~nm}$, depending on design constraints. The $\mathrm{Cr}$ layer is usually left on the templates until after the cutting process, which is described below; this facilitates template pattern recognition, and minimizes mistakes in cutting.

Template fabrication schemes have also been investigated which embed a transparent conducting oxide into the final template. The addition of a blanket conducting layer subverts charging problems associated with SEM inspection of the final solid glass templates. One such scheme involves coating the fused silica plate with a film of indium tin oxide (ITO), which is transparent and conducting. 9, 12 The ITO film is then capped with a film of deposited $\mathrm{SiO}_{2}$, followed by e-beam resist The resist is patterned using an electron beam tool, and chemically developed. The patterned resist is used as an etch mask to pattern the underlying $\mathrm{SiO}_{2}$ film, and finally stripped. The resulting template consists of patterned $\mathrm{SiO}_{2}$ features resting on a blanket film of ITO on fused silica substrate. The $\mathrm{SiO}_{2}$ features will define the imprinted features in the SFIL process, while the ITO film is transparent to allow the SFIL process exposure and conducting to allow SEM inspection of the template.

Another template scheme consists of spinning a film of hydrogen silsesquioxane (HSQ) on the ITO layer; the HSQ is directly written with e-beam lithography. ${ }^{13}$ In its cured state, HSQ becomes a durable oxide making it a very convenient material for direct patterning of SFIL template relief structures. One benefit of this scheme is that it eliminates the etching processes associated with other template fabrication methods.

Each imprint template is 1 -in by 1 -in, which is larger than the largest die size predicted in the SIA Roadmap. ${ }^{1}$ Several templates can be made on each 6-in substrate. The individual templates can be cut out using a variety of methods, such as a precision wheel saw, water knife, or cutting laser. Regardless of the cutting method used, the final template dimensions must meet the constraints of the imprint equipment. After cutting, the $\mathrm{Cr}$ is removed typically by immersion in a solution of ceric ammonium nitrate, and the surface is cleaned and treated as described below.

\subsection{Surface Treatment}

It is imperative, following exposure, that the polymerized etch barrier adhere to the underlying transfer layer, and release easily and completely from the template. The successful release of the imprint template from the imprinted polymer depends in part on the surface energies of the template and the imprinted polymer. A fluoroalkyltrichlorosilane precursor is used to form such a monolayer on the template surface to promote this release. ${ }^{3,14}$

In one set of experiments the quartz templates were cleaned in an acetone ultrasonic bath, followed by $\mathrm{O}_{2} \mathrm{RIE}$ at $50 \mathrm{~W}, 10 \mathrm{sccm} \mathrm{O}_{2}, 20$ $\mathrm{mTorr}$ for $10 \mathrm{~min}$ to remove organic contaminants on the surface. The clean templates were then treated with a release agent, tridecafluoro-1,1,2,2tetrahydrooctyltrichlorosilane (Gelest), by vapor exposure at $1 \mathrm{~atm}$ total pressure (precursor plus $\mathrm{N}_{2}$ ) for $90 \mathrm{~min}$ at room temperature, and annealed at $100^{\circ} \mathrm{C}$ for $15 \mathrm{~min}$. This surface treatment reaction 
has yielded surface energies in the neighborhood of 12 dynes $/ \mathrm{cm} .^{14}$

The surface treatment must maintain its release characteristics through thousands of imprints in a manufacturing process. Preliminary results indicate that the current technique could provide films with the required durability. ${ }^{14}$ We have seen no evidence of catastrophic loss of release function, and work is currently underway to quantify film durability for a variety of surface treatment conditions.

\subsection{Etch Barrier}

The etch barrier material is subject to several design constraints. The etch barrier liquid must be dispensable from an automatic fluid dispense system, and must not change significantly in composition between dispensing and imprinting by, e.g., component evaporation. It must be readily displaced during the imprint step and photopolymerize rapidly during exposure. Shrinkage due to polymerization must be controlled. The polymer must release from the template while adhering to the transfer layer, and it must exhibit sufficient rigidity to avoid feature collapse It must exhibit some level of temperature stability to withstand the etching temperatures, and it must exhibit sufficient etch selectivity during the $\mathrm{O}_{2}$ RIE step to allow for high aspect ratios to be generated in the transfer layer.

The SFIL process relies on photopolymerization of a low viscosity, acrylatebased solution. Acrylate polymerization is known to be accompanied by volumetric shrinkage that is the result of chemical bond formation. Consequently, the size, shape, and placement of the replicated features may be affected. Volumetric shrinkage was found to be less than $10 \%(\mathrm{v} / \mathrm{v})$ in most cases. ${ }^{15,16}$

Finite element modeling (FEM) results indicate that no significant pattern placement distortion will result as a consequence of densification. Additionally, the sidewall angle is predicted to be close to $90^{\circ}$ for high aspect ratio features, and will approach $80^{\circ}$ for low aspect ratio features, using the worst-case model parameter of $17 \%(\mathrm{v} / \mathrm{v})$ densification. ${ }^{15}, 16$

The current etch barrier liquid is a multicomponent solution, and has been described previously. The silylated monomer provides etch resistance in the $\mathrm{O}_{2}$ transfer etch. Crosslinker monomers provide thermal stability. to the cured etch barrier and also improve the cohesive strength of the etch barrier. Organic monomers serve as mass-persistent components and lower the viscosity of the etch barrier formulation. The photoinitiators dissociate to form radicals upon UV irradiation, and these radicals initiate polymerization.

In separate studies of various acrylate and methacrylate monomers, the effect of fluence was examined using IR to follow the isolated $\mathrm{C}=\mathrm{C}$ stretching absorbance peak at $1640 \mathrm{~cm}^{-1}$ and revealed nearly complete conversion with less than $30 \mathrm{~mJ} / \mathrm{cm}^{2}$. Further information on UV curing of acrylate coatings can be found in works by Decker ${ }^{17}$ and Kloosterboer. ${ }^{18-20}$

\subsection{Transfer Layer}

The transfer layer is an organic film that can be tailored in thickness and properties for specific application, but must follow certain guidelines. The transfer layer can not be soluble in the liquid etch barrier, and must adhere well to the cured etch barrier. The transfer layer must remain intact after exposure. There should be at least modest etch selectivity between the etch barrier and the transfer layer in order to obtain aspect ratio magnification. These and other issues are being considered as materials development continues.

We are currently using a commercial bottom anti-reflection coating as a transfer layer, but have experimented with negative resists and other spun polymers as well. 3,7

\section{Results}

Results from our collaboration with Agilent Technologies were very encouraging. The UTAgilent process was slightly different than that shown in Figure 1 in that the imprint template was compliant, and the imprinted material was more viscous. The collaboration, however, helped to demonstrate capabilities of the SFIL process. The goal of the collaboration was to demonstrate the ability of SFIL to pattern over topography. This would be especially important in, for example, patterning steps during front-end processing where topographies cannot be polished smooth. Figure 2 shows a SEM image of etched polymer features on a patterned $\mathrm{Si}$ substrate. 


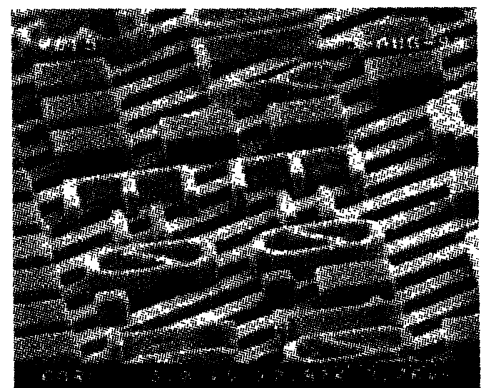

Figure 2. SEM image demonstrating the capability of SFIL to pattern over existing topography.

We have also undertaken an effort to quantify defectivity as a function of repeated imprints. Early results were very encouraging, showing no catastrophic generation or propagation of defects. Figure 3 shows a micrograph of an imprint template before installation in the SFIL stepper, and after only two imprints. The handling of the template during installation in the current operating procedure can impart some contamination on the template, but imprinting actually cleans away that contamination.
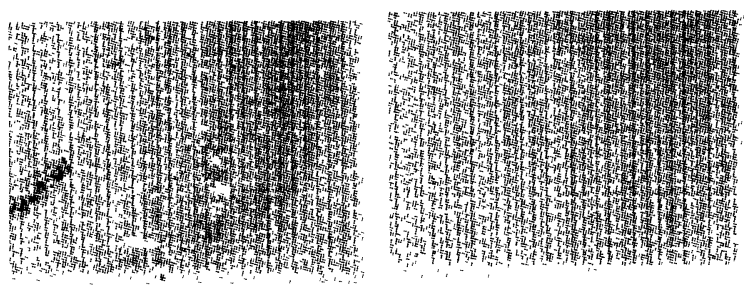

Figure 3. Images of an imprint template before (left) and after (right) two imprints. Template contamination is removed during imprinting.

Recent etch transfer results demonstrate the process capabilities. Figure 4 shows crosssectional SEM images of $500 \mathrm{~nm}$ and $80 \mathrm{~nm}$ features taken from the same imprint field, demonstrating the tremendous process latitude of the SFIL process
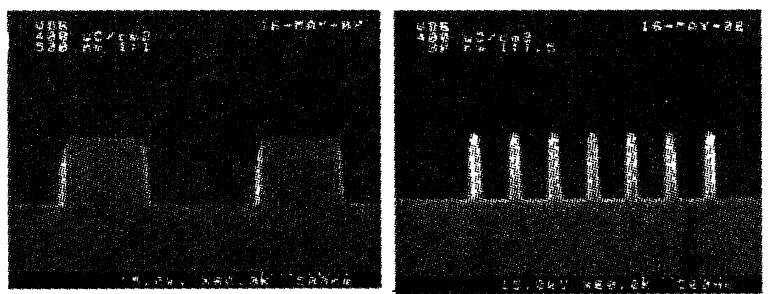

Figure 4. SEM images of $500 \mathrm{~nm}$ (left) and $80 \mathrm{~nm}$ (right) features etched through the residual etch barrier and transfer layer to the $\mathrm{Si}$ substrate.

\section{Summary}

The goal of the SFIL development program is to enable replication of sub- $100 \mathrm{~nm}$ features at room temperature with minimal applied pressure. We believe the use of low viscosity, photopolymerizable materials will enable SFIL to achieve the throughput required for use in the microelectronics industry. Additionally, the rigid, transparent imprint template used in SFIL enables a precision in overlay alignment that is difficult to achieve in other imprint schemes.

The design of SFIL materials is consistent with the requirements of high-volume manufacturing. SFIL has been developed to allow the process to meet the throughput, resolution, and die size requirements outlined in the SIA International Technology Roadmap for Semiconductors. We have shown SFIL to be a high-resolution technique capable of patterning a wide variety of substrates at room temperature, under low applied pressure, in a reliable, cost-effective manner.

\section{Acknowledgements}

The authors thank Motorola Labs, International SEMATECH, Brewer Science, DPI-RTC, Agilent Technologies, IBM, Ultratech Stepper, ETEC, 3M, NRL, and Compugraphics for generous gifts and technical consultation. Special thanks to Matthew Colburn, Byung Jin Choi, and Mario Meissl for their help in developing the SFIL process and equipment, Andrew Jamieson, David Stark, and Daniel Miller for their help with the current etching efforts, Michael Rich and Georgia Rich for ARC processing. We gratefully acknowledge the financial support of DARPA (MDA972-97-10010) and SRC (96-LC-460).

\section{References}

1. SIA, 2001: available at http://public.itrs.net/Files/2001ITRS/Home.ht $\mathrm{ml}$.

2. Chou, S.Y., P.R. Krauss, and P.J. Renstrom, J. Vac. Sci. Tech. B, 1996. 14(6): p. 4129.

3. Colburn, M., et al., Proc. SPIE: Emerging Lithographic Technologies III, 1999. 3676(I): p. 379.

4. Haisma, J., et al., J. Vac. Sci. Tech. B, 1996. 14(6): p. 4124. 
5. Xia, Y. and G.M. Whitesides, Angew. Chem. Int. Ed. Engl., 1998. 37: p. 550.

6. Thompson, L.F., C.G. Willson, and M.J. Bowden, Introduction to Microlithography. 2nd ed. 1994, Washington, D.C.: ACS.

7. Colburn, M., et al., Proc. SPIE: Emerging Lithographic Technologies IV, 2000. 3997: $p$. 453.

8. Ruchoeft, P., et al., J. Vac. Sci. Tech. B, 1999. 17(6): p. 2965.

9. Bailey, T.C., et al., Microelectron. Eng., 2002: accepted for publication.

10. Resnick, D.J., et al., Proc. SPIE: Emerging Lithographic Technologies VI, 2002. 4688: submitted.

11. Choi, B.J., et al., Proc. SPIE: Emerging Lithographic Technologies V, 2001. 4343: p. 436.

12. Dauksher, W.J., et al., J. Vac. Sci. Tech. B, 2002. 20(6): submitted.
13. Mancini, D.P., et al., J. Vac. Sci. Tech. B, 2002. 20(6): submitted.

14. Bailey, T., et al., J. Vac. Sci. Tech: B, 2000. 18(6): p. 3572.

15. Colburn, M., et al., J. Vac. Sci. Tech. B., 2001. 19(6): p. 2685.

16. Colburn, M.E., Step and Flash Imprint Lithography: A Low-Pressure, RoomTemperature Nanoimprint Lithography. Department of Chemical Engineering; Ph.D. Thesis. 2001, Austin, TX: The University of Texas at Austin.

17. Decker, C., Prog. Polym. Sci., 1996. 21: p. 593.

18. Kloosterboer, J.G. and G.J.M. Lippits, J. Imaging Science, 1986. 30(4): p. 177.

19. Kloosterboer, J.G. and G.F.C.M. Lijten, Polymer, 1987. 28(7): p. 1149.

20. Kloosterboer, J.G., G.F.C.M. Lijten, and C.P.G. Zegers, Polym. Mater. Sci. Eng., 1989. 60: p. 122. 